\author{
Military Technical College \\ Kobry El-Kobba \\ Cairo, Egypt
}

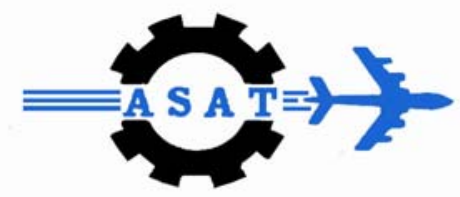

12-th International Conference

on

Aerospace Sciences \& Aviation Technology

\title{
SINGLE-TRANSVERSE-MODE BIPOLAR CASCADE LASERS WITH THICK QUANTUM WELLS AND NO OPTICAL CONFINEMENT LAYERS
}

\author{
Tarek A. Ramadan*
}

\begin{abstract}
The use of thick quantum wells with no optical confinement layers allows designing single-transverse-mode bipolar cascade lasers with high external differential quantum efficiencies. By controlling the number of wells the available laser power is maximized.
\end{abstract}

\section{INTRODUCTION}

The recycling of carriers by tunnel junctions allows designing bipolar cascade lasers (BCLs) with high external differential quantum efficiencies (DQEs) that increase with increasing the number of active regions, $M$. Thus, providing an additional gain factor which may be utilized in different applications such as direct modulation of optical carriers by analog signals. At the same time the requirement of single-transverse-mode (STM) operation of BCLs sets up an upper bound on $M$, which limits the DQE. This bound is not high (e.g. 2 or 3) [1], mainly due to the non-zero thickness of the optical confinement layers (CLs), which are used for the purpose of maintaining sufficient electron-photon interaction and avoiding large penalty on the threshold current.

In this work the DQE of the STM-BCL is maximized by eliminating the CLs and maximizing $M$. It is shown that this elimination does not sacrifice the optical power confinement, $\Gamma$, which also increases by increasing $M$. Thick quantum wells are used to ensure efficient collection of carriers in the absence of CLs.

\section{THEORY}

The steady state solution of simplified rate equations was used to derive the following expression of the DQE of a BCL,

$$
\eta=\left(M \eta_{s}\right)\left[(\Gamma / M) /\left(N t_{w} d / w\right)\right] .
$$


* Department of Physics, Faculty of Science, Kuwait University, P.O. Box 5969, Safat-13060, Kuwait (e-mail: tar10@kuc01.kuniv.edu.kw)

Here, $\eta_{s}$ is the conventional DQE of a single active region, $N$ is the number of quantum wells in each active region, $t_{w}$ is the thickness of each well, $d$ is the lateral width of the active regions, and $w$ is the power-equivalent modal spot size. The quantity between brackets represents the deviation of recycling efficiency, $\eta_{r}\left(\equiv \eta / \eta_{s}\right)$, from linearity with increasing $M$. It equals the ratio between the average optical confinement, $\Gamma / M$, to the maximum possible optical confinement, $N t_{w} d / w$, of each active region. In a broad-area $\mathrm{BCL}, N t_{w} d / w \rightarrow N t_{w} / w_{\perp}$, where $w_{\perp}$ is the power-equivalent modal width in the direction normal to the substrate.

An analytical expression of the upper bound on $M$ has been derived by invoking the root-mean-square (rms) index approximation and applying the single array-mode condition [2]. This bound is given by,

$$
\hat{M}=\left\lfloor\pi / \cos ^{-1}(\cos (T)-(S / 2) \sin (T))\right\rfloor,
$$

where $\lfloor u\rfloor$ denotes the floor of $u, T \equiv k_{o} t \sqrt{\tilde{n}_{1}^{2}-n_{2}^{2}}$, and $S \equiv k_{o} s \sqrt{\tilde{n}_{1}^{2}-n_{2}^{2}}$. Here, $t$ is the thickness of a single active region, $s$ is the separation between successive regions, $k_{o}\left(=2 \pi / \lambda_{o}\right)$ is the free-space propagation constant, $\tilde{n}_{1}$ is the rms approximation of the core index, and $n_{2}$ is the cladding refractive index. According to (2), $\hat{M}$ increases by decreasing $t, \mathrm{~s}, \tilde{n}_{1}$, and/or increasing $n_{2}$. The index $\tilde{n}_{1}$ decreases as $N$ decreases, which suggests using a single quantum well (SQW) instead of multiple QWs in each active region for the purpose of maximizing $\hat{M}$.

\section{CONFINEMENT LAYERS}

A planar $\mathrm{Al}_{x} \mathrm{Ga}_{1-x} \mathrm{As}$ design example of a $\mathrm{BCL}$ is used to demonstrate the effect of minimizing the thickness $t_{C}$ of the CLs on $\eta_{r}$ and $\Gamma$, under the condition, $M=\hat{M}$. Each active region of the specific design consists of a separate-confinement heterostructure $(\mathrm{SCH})$ with a SQW of thickness, $t_{w}=20 \mathrm{~nm}$. This relatively large thickness of the $\mathrm{QW}$ is chosen to ensure efficient collection of carriers in the absence of CLs (i.e. as $t_{C} \rightarrow 0$ ) [3]. The values of $x$ are $0.4,0.2$, and 0 in the cladding, barrier, and QW layers. The separation between successive active regions is fixed at a minimum value which is limited by the thickness of the tunnel junctions. This minimum value is chosen to be $s=100 \mathrm{~nm}$ for $50 \mathrm{~nm}$-thick tunnel junctions. The thickness $t_{c}\left(\equiv t-t_{w}\right)$, decreases by decreasing $t$. It becomes zero when $t=t_{w}$. Fig.1 shows $\eta_{r}$ and the corresponding $\hat{M}$ versus $t_{C}$. In the limit as $t_{C} \rightarrow 0, \eta_{r} \rightarrow 4.70$ and $\hat{M} \rightarrow 6$. Fig. 2 compares $\Gamma$ versus $t_{C}$ 
for this $\mathrm{BCL}$ (with $M=\hat{M}$ ) to that of the corresponding conventional $\mathrm{SCH}$ laser with a single active region $(M=1)$. While in the latter case $\Gamma$ reaches a minimum value of 0.02 , in the former case it reaches a maximum value of 0.14 , as $t_{C} \rightarrow 0$. This fundamental difference between the two cases is mainly due to the corresponding increase in $M$ of the $\mathrm{BCL}$ as $t_{C} \rightarrow 0$. These results suggest eliminating the conventional CLs and maximizing $M$ in the design of BCLs.

\section{STM RIDGE_WAVEGUIDE BCL}

The above planar design of $B C L$ (with $M=6$ and no CLs) was used as the vertical structure of a STM ridge-waveguide BCL as in [2], with a cover layer thickness of $1 \mu \mathrm{m}$ and $0.2 \mu \mathrm{m}$ in the ridge and slab regions, respectively. The ridge width, $d=2.9 \mu \mathrm{m}$, was chosen using the effective index method to maximize the lateral modal confinement under STM condition.

As a preliminary step to compute the threshold current density, $J_{t h}$, the gain of a SQW was computed as in [4], with an intraband relaxation time of $50 \mathrm{fs}$. Because of the relatively large well depth that results from eliminating the CLs, the nonparabolicity of the conduction band was included in these computations through an energy-dependent effective mass, as in [5]. The peak-gain-current relation was used to compute $J_{\text {th }}$ and the threshold current $I_{\text {th }}$ as a function of the cavity length, $L$. In these computations, the internal quantum efficiency of a single active region is 0.8 , the laser facets are uncoated while the optical propagation loss coefficients in the QW, tunnel, and cladding regions are $20 \mathrm{~cm}^{-1}, 50 \mathrm{~cm}^{-1}$, and $10 \mathrm{~cm}^{-1}$, respectively. The results show that at $J_{t h}=400 \mathrm{~A} / \mathrm{cm}^{2}$, $L=195 \mu \mathrm{m}, I_{t h}=2.3 \mathrm{~mA}$ and $\eta=2.86$. The corresponding $w=1.7 \mu \mathrm{m}^{2}$ (with $w_{\perp}=0.65 \mu \mathrm{m}$ ) leads to an available laser power of $60 \mathrm{~mW}$, assuming a catastrophic damage power density of $35 \mathrm{~mW} / \mu \mathrm{m}^{2}$ outside the laser cavity.

\section{TRADEOFF BETWEEN DQE AND AVAILABLE LASER POWER}

Unlike $\eta_{r}$ which is maximized by minimizing $s$ and maximizing $M$, the power equivalent modal width $w_{\perp}$ (and consequently the available laser power) is maximized by maximizing $s$ and minimizing $M[1,2]$. This tradeoff is verified by computing $\eta_{r}$ and $w_{\perp}$ for the planar design of section III (with no CLs) as a function of $M$ under minimum and maximum separation $s$ between the active regions. As before, the minimum $s=100 \mathrm{~nm}$ while the maximum $s$ is determined from the STM condition [2]. The results of computations in Fig. 3 show that by reducing $M$ and maximizing $s$, high values of $w_{\perp}$ are obtained (e.g. $w_{\perp}=1.5 \mu \mathrm{m}$ at $M=2$ ). However, this minimization of $M$ sacrifices the optical power confinement and increases the penalty on the threshold current. In order to estimate the threshold current requirements, both of the threshold current per unit stripewidth $\bar{I}_{t h}\left(\equiv I_{t h} / d\right)$ and $J_{t h}$ were computed as a function of cavity length, $L$, at different $M$ 
and maximum s. Fig. 4 shows $L$ and $\bar{I}_{\text {th }}$ at $J_{\text {th }}=400 \mathrm{~A} / \mathrm{cm}^{2}$, as a function of $M$. At $M=3$ and maximum separation $s=0.45 \mu \mathrm{m}$ the values of $L=1.1 \mathrm{~mm}$ and $\bar{I}_{t h}=4.4 \mathrm{~mA} / \mu \mathrm{m}$ while $w_{\perp}=1.1 \mu \mathrm{m}$ and $\eta_{r}=2.3$.

The above planar design (with $M=3$ and no CLs) was used as the vertical structure of a ridge waveguide, as in section 4 . The cover layer thickness is chosen to be $1.5 \mu \mathrm{m}$ and $0.2 \mu \mathrm{m}$ in the rib and slab regions of this waveguide. The ridge width $d=3.3 \mu \mathrm{m}$ maximizes the lateral modal confinement under STM condition. The corresponding $w=3.2 \mu \mathrm{m}^{2}$ (with $w_{\perp}=1.1 \mu \mathrm{m}$ ) leads to an available laser power of $112 \mathrm{~mW}$ while $I_{\text {th }}=14.5 \mathrm{~mA}$ and $\eta=1.39$.

\section{REFERENCES}

[1] T. A. Ramadan, "High-power expanded-core-waveguide edge-emitting bipolar cascade lasers," in Conference on Lasers and Electro-Optics, technical digest (Optical Society of America, 2006), paper JWB9.

[2] T. A. Ramadan, "Expanding the core: A new approach for the design of single-mode waveguides," IEEE J. Lightw. Technol. 23, 3843-3856 (2005).

[3] H. Shichijo, R. M. Kolbas, N. Holonyak, Jr., R. D. Dupuis, and P. D. Dapkus, "Carrier collection in a semiconductor quantum well," Solid State Commun. 27, 1029-1032 (1978).

[4] S. L. Chuang, Physics of Optoelectronic Devices (Wiley, 1995), Chap. 9.

[5] R. Nagarajan, T. Kamiya, and A. Kurobe, "Band filling in GaAs/AlGaAs multiquantum well lasers and its effect on the threshold current," IEEE J. Quantum Electron. 25, 1161-1170 (1989). 


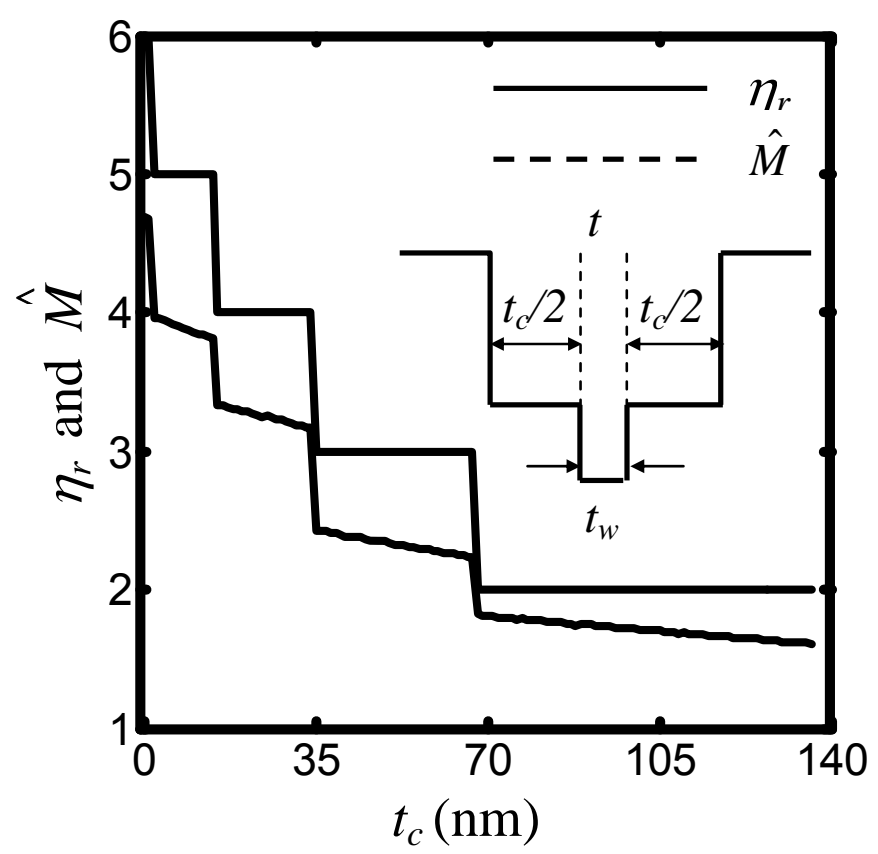

Fig.1. $\eta_{r}$ (solid line) and $\hat{M}$ (dashed line) versus $t_{c}$. The inset shows $t_{c}, t$, and $t_{w}$ on a schematic of the conduction band of a single active region.

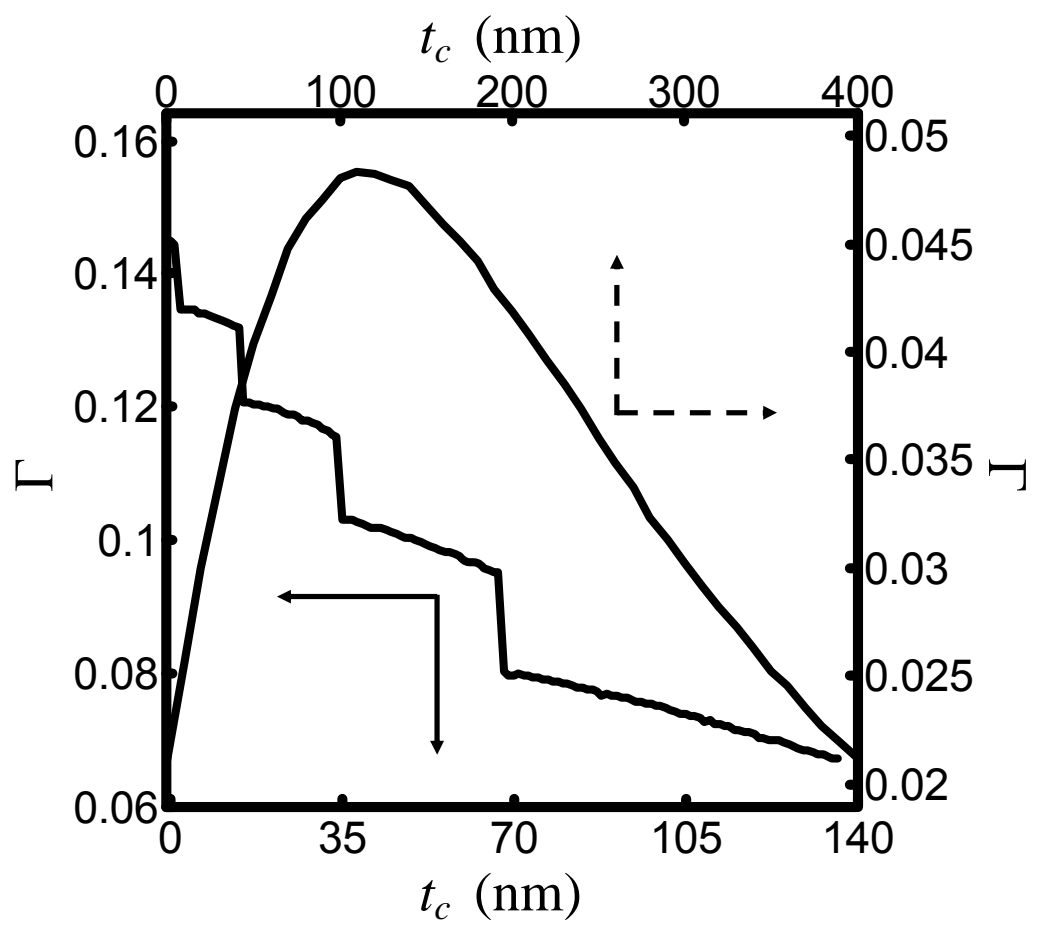


Fig.2. $\Gamma$ of the $\mathrm{BCL}$ with $M=\bar{M}$ (solid line) and that of the corresponding conventional $\mathrm{SCH}$ laser with $M=1$ (dashed line) versus $t_{c}$.

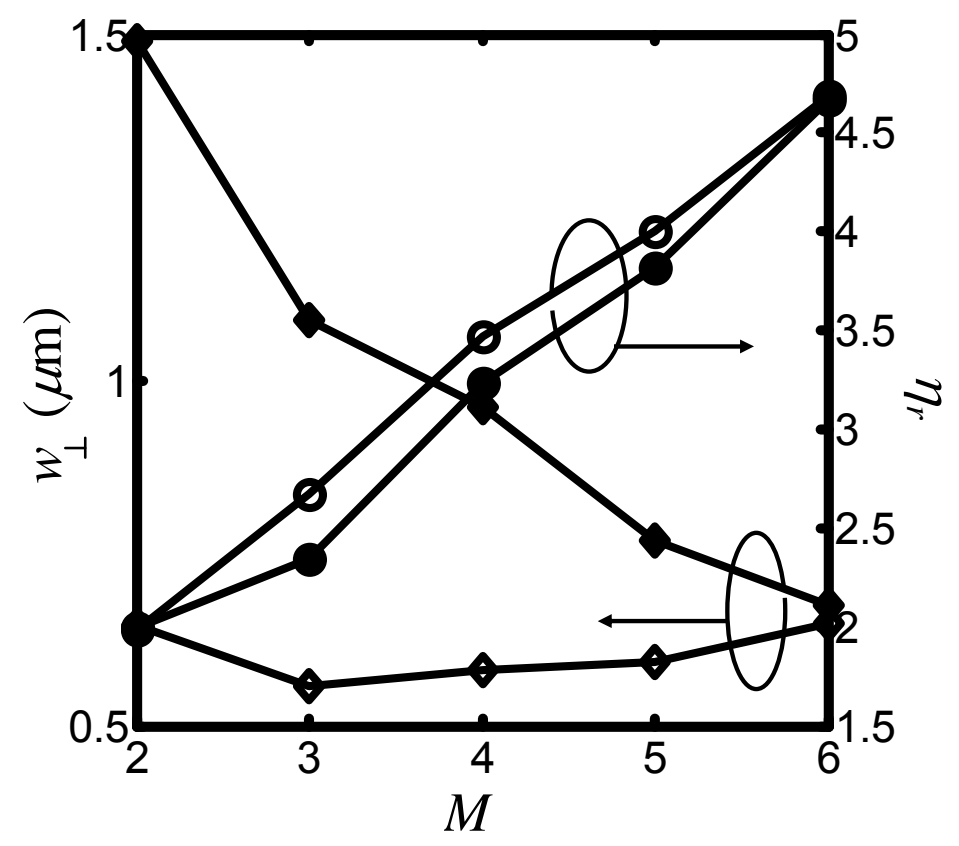

Fig.3. $\eta_{r}$ (circles) and $w_{\perp}$ (diamonds) versus $M$ under maximum (closed) and minimum (open) separation $s$ between the active regions. The dashed lines have no meaning. They connect relevant data points to clarify their dependence on $M$.

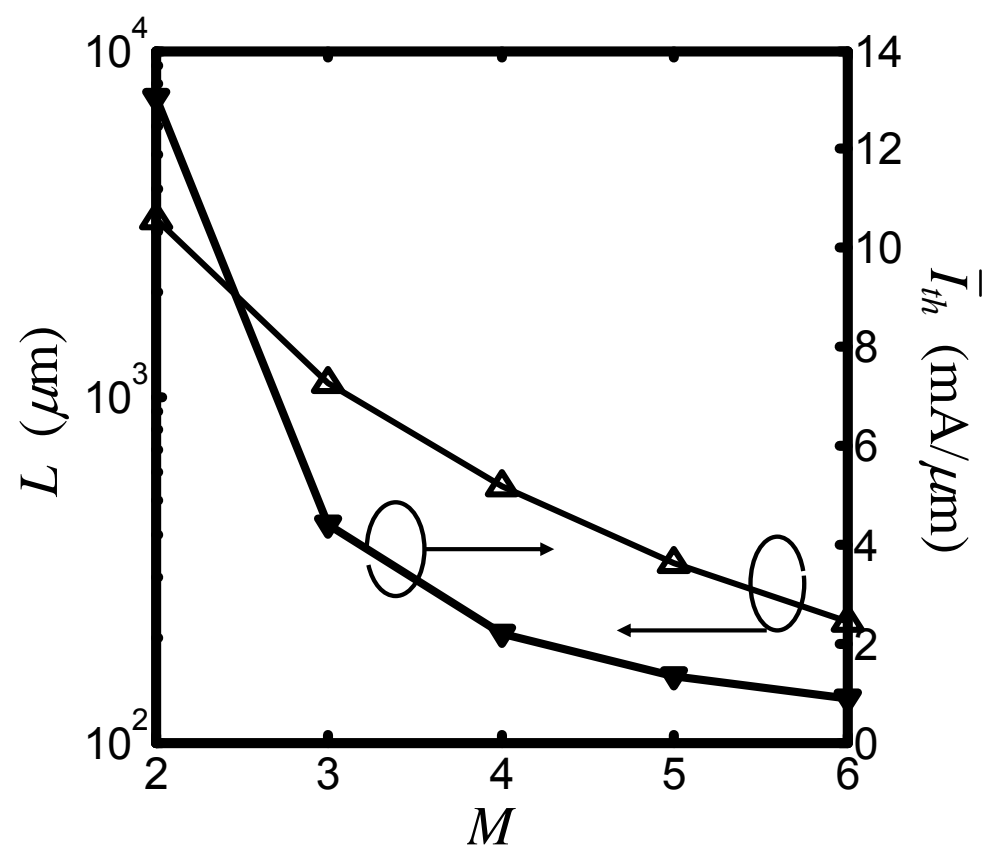


Fig.4. $L$ (up triangles) and $\bar{I}_{\text {th }}$ (down triangles) versus $M$ at $J_{t h}=400 \mathrm{~A} / \mathrm{cm}^{2}$. The dashed lines have no meaning. They connect relevant data points to clarify their dependence on M. 